\title{
Key distance effects on perceived harmonic structure in music
}

\author{
CAROL L. KRUMHANSL \\ Cornell University, Ithaca, New York \\ JAMSHED BHARUCHA \\ Harvard University, Cambridge, Massachusetts \\ and \\ MARY A. CASTELLANO \\ Cornell University, Ithaca, New York
}

\begin{abstract}
Two experiments provide convergent evidence for the hypothesis that listeners interpret chords in terms of their harmonic functions in a system of interrelated keys. The perceived associations between chords undergo significant changes depending on the broader tonal framework in which they are embedded. Three independent context effects are identified, and their magnitude is found to be a systematic function of the distance between the context key and the key (or keys) of which the chords are members. In the first experiment, listeners rate how musically related one chord is to a second chord; the chords are those that function within two maximally distant major keys (C major and F" major). All possible chord pairs from this set are presented in each of three context keys: $G$ major (which is close to $C$ major and distant from $F$ " major), A major (which is moderately distant from both $\mathrm{C}$ major and F " major), and B major (which is close to F" major and distant from $C$ major). The second experiment measures recognition memory for the same chords embedded in tonal sequences in C, G, A, or B major keys, or random sequences. In the two experiments, the distance between the context key and the key of the chords on the circle-of-fifths affects: (1) the probability that a repeated chord is correctly recognized, (2) the strength of association between chords from the same key measured in terms of both confusion errors and direct relatedness judgments, and (3) asymmetries in confusion errors and relatedness judgments when the chords are in different keys. Perceived harmonic relations are thus found to be strongly context dependent, but the context effects are lawful functions of interkey distance. We conclude that listeners possess a highly articulated system of knowledge about the harmonic functions of chords in musical keys and an appreciation of musical structure at the level of abstract tonal centers.
\end{abstract}

When listening to music, we do not hear the individual tones and chords as disconnected units. Instead, they are perceived in relation to one another, and the sense of a coherent and well-organized whole emerges. This process depends on the fact that the musical elements (tones and chords) and their ordering are not random but conform to patterns characteristic of our musical culture. Through experience with music, listeners have presumably abstracted and internalized certain underlying regularities. This system of knowledge may be employed during listening to emphasize particular elements and relations between elements, to give rise to expectations as to

This research was supported by a grant to the first author from the National Science Foundation (BNS 081-03570). The helpful comments of two anonymous reviewers on an earlier draft are gratefully acknowledged. Reprint requests may be addressed to: Carol L. Krumhansl, Department of Psychology, Uris Hall, Cornell University, Ithaca, New York 14853. what is likely to follow, and to enable the listener to apprehend the more global organization of the composition. Our research focuses on the question of how the listener's knowledge of musical structure is represented and engaged during music perception.

Music theorists describe the underlying regularities in traditional Western music in terms of tonality or key. Two aspects of this structural description will be important for the present empirical investigation. The first is key membership, which specifies the tones and chords that are typically employed in a musical passage written in a particular key. These elements conform to a fixed pattern of interval relations centered around a single tone, called the tonic of the key. Previous empirical investigations, to be described briefly later, indicate that listeners familiar with tonal music have internalized this aspect of musical structure. The second property identified by music theorists is key distance, which specifies the varying strength of association between different musical 
keys. Certain pairs of keys are considered to be closely related to each other, and others less so. Thus, music theorists ascribe considerable structure to the set of abstract tonal centers or keys. The internalization of musical structure at this level has found some support in previous studies, and will be investigated further in the present article.

The specific issue addressed here was whether key distance affects perceived harmonic relations, that is, associations between elements at the level of chords. We investigated how the internal representation of harmonic structure, measured in terms of direct relatedness judgments and memory confusions, is influenced by the context key in which the chords are embedded. If these interelement associations are found to vary systematically with the distance between the context key and the key (or keys) in which the chords function, this would strongly support the view that listeners interpret chords in terms of their functions within keys and, moreover, have internalized relations between keys, as described in the music-theoretic account of key distance.

\section{Key Membership}

Every key is associated with a subset of pitches that constitute the scale of the key; usually these form a major or minor diatonic scale. Most tones of a composition, particularly those that are frequent and rhythmically stressed, are drawn from this subset. Much of Western music, however, is constructed of chords, either sounded as simultaneous tones or implied by the succession of tones. To each key there corresponds a subset of the possible chords; the basic set of harmonies of a key are the triads (with three different tones separated by thirds) constructed from the tones of the musical scale. Roman numerals are often used to designate the position of the root of the triad in the scale; for example, I designates the triad built on the first step of the scale. This account, based on analyses of musical compositions, suggests that an essential organizing property of tonal music is the sounding of tones and chords that are members of a musical key.

Previous empirical studies indicate that listeners are sensitive to key membership-that is, the functioning of certain tones and chords in musical keys. In one study, Bharucha and Krumhansl (in press) presented all possible pairs of chords from two distantly related keys and asked listeners to rate how closely related the first chord was to the second chord of the pair. These relatedness judgments were analyzed using multidimensional scaling (Kruskal, 1964; Shepard, 1962), which produces a spatial configuration of points such that interpoint distances in that spatial configuration are monotonically related (as much as possible) to the relatedness judgments. In that analysis, the chords of the two keys separated into two distinct clusters, representing the fact that the chords of one key were perceived as more musically related to one another than they were to chords of the other key. Thus, at least for chords, key membership is an important determinant of the perceived strength of association between musical elements. This is true even when the chords were presented in isolation, without an experimentally instantiated tonal context. When a context key was indicated explicitly by sounding a strong chord cadence in that key, key membership became an even stronger factor in that study. The effect of introducing a tonal context was to draw together the chords of the context key (which, in music theory, are called diatonic) more closely than when no context was provided. The relations among nondiatonic chords (those not in the basic set of harmonies of the context key) were weakened by this manipulation. That study also contained a parallel experiment using recognition memory for chord sequences, which again pointed to the importance of key membership. When the sequences suggested an underlying key, the substitution of one chord within the key by another chord within that key was detected less frequently than were changes outside the key.

Key membership also appears as a factor for single tones, which, in isolation, have more ambiguous tonal interpretations than chords. In a scaling study of tones within an octave range, Krumhansl (1979) found that tones contained within the scale of the experimentally defined context key were perceived as more musically interrelated than those outside the key. Krumhansl and Keil (1982) observed a similar distinction between diatonic and nondiatonic tones in judgments about short melodies given by children of elementary school age. A number of studies (Cuddy, Cohen, \& Miller, 1979; Dowling, 1978; Krumhansl, 1979) have shown that key membership also affects recognition memory for tones embedded in tonal sequences. Tones within the key are more frequently confused with other tones in the key than with nondiatonic tones. In the scaling study of single tones (Krumhansl, 1979), an additional factor emerged. The tones judged as most closely interrelated were those that form the tonic triad chord, the most harmonically significant chord of the key. The special functions of these tones were also observed in two other studies. In these, the listeners were asked to rate how well single tones fit with a number of keydefining elements, such as scales, chords, and chord cadences (Krumhansl \& Kessler, 1982; Krumhansl \& Shepard, 1979). Highest ratings were given to the tones of the major triad chord, intermediate ratings were given to the other scale tones, and lowest ratings were given to the nondiatonic tones. At the level of single tones, then, membership in both the key and in the tonic triad chord appear to be contained in the internal representation of pitch relationships. 
These results have led us (Krumhansl, Bharucha, \& Kessler, 1982) to propose a three-leveled structure: The lowest level comprises single tones, the intermediate level, chords, and the highest level, the different tonal centers or keys. The perceived interelement associations at the level of chords are presumed to be mediated, at least in part, by their functions within musical keys, particularly the key established by the experimental context. Intertone relations are assumed to be determined by their membership both in the context key and in the chords with the greatest functional significance in that key. Because the perceived associations between tones and between chords depends significantly on their relation to the tonal context in which they are embedded, structure at the level of abstract tonal centers seems a necessary component of the internal representation of musical knowledge. Invariant relations are unlikely to be found at the level of tones and chords, but may emerge for abstract tonal centers.

\section{Key Distance}

Music theorists have described structure at the level of abstract tonal centers in terms of the different degrees of relatedness between the musical keys. Because this concept of key distance is central to the experiments to be reported, it will be described here in some detail. Key distance is important for characterizing more global aspects of musical organization, that is, the relations among different sections of a musical composition. Musical compositions frequently contain modulations (changes) between keys, with the distance between keys approximately correlated with the ease of modulation between them. These shifts are used to provide contrasts of varying degrees and highlight major boundaries within the composition. Moreover, consecutive movements of a musical composition may be held together by nothing other than the proximity of their keys.

Music theory identifies three features that give the same measure of distance between two major keys: the number of tones shared by the scales of the keys, the difference in the number of sharps or flats in the key signatures, and the distance around the circle of fifths, which is shown in Figure 1. Each key is flanked by the keys whose tonics are an interval of a perfect fifth above and below the tonic of that key. (The tonic is the first note of the scale and gives the name to the key.) This fifth relation continues to define interkey distance until, after moving six positions around the circle of fifths, the most distantly related major key is reached in both directions. So major keys are represented as points around a circle. Each major key is also closely associated with two minor keys: its relative minor (which is built on the sixth note of the major scale and whose scale in its
CIRCLE OF FIFTHS FOR MAJOR KEYS

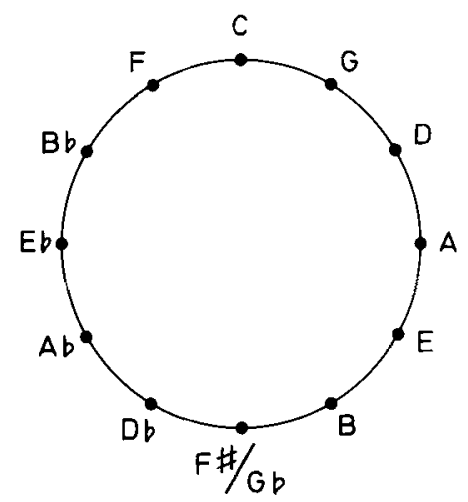

Figure 1. The circle-of-fifths for the 12 major keys. The tonics of adjacent keys are separated by an interval of a perfect fifth. Adjacent keys share all but one scale tone, and differ by one sharp or fiat in their key signatures.

natural form contains the same pitches as the major scale) and its parallel minor (which is the minor key with the same tonic as the major key). Because of these relative and parallel relations among major and minor keys, the total set of major and minor keys can no longer be represented simply by the circle of fifths. However, the experiments to be reported here employ only major key contexts, so the circle of fifths is a sufficient representation of the musictheoretic account of key distance for the present purposes.

Krumhansl and Kessler (1982) recently found empirical support for this music-theoretic description of interkey distance and provided a spatial representation of musical keys that included both major and minor keys. This spatial representation was obtained using a tone-profile technique. This general technique was introduced in an earlier study (Krumhansl \& Shepard, 1979) in which listeners heard an incomplete scale sequence followed by any one of the pitches of the chromatic scale in the next octave range. The task was to rate how well this last tone completed the scale sequence. Listeners with a moderate-to-high level of music training produced response profiles containing considerable structure, suggesting that ratings of this sort might be used to determine distances between keys.

In the Krumhansl and Kessler (1982) study, this tone-profile technique was applied to a variety of musical elements (scales, chords, and chord cadences) that are unambiguous indicators of musical keys. The obtained profiles were averaged for the elements defining the same key, and were then shifted to different reference pitches (tonics) and intercorrelated to produce a measure of interkey distance. Multidimensional scaling of the correlations 
produced a very regular spatial representation of the 24 major and minor keys. When only the 12 major keys were included, the circle of fifths (shown in Figure 1) was obtained. Adding the minor keys necessitated the introduction of two more dimensions. In the four-dimensional solution, the first two dimensions again contained the circle of fifths, and the second two dimensions accommodated the parallel and relative relations between major and minor keys. This analysis of the profile correlations located the points for the major and minor keys on the surface of a torus in four dimensions in a way that confirmed the qualitative account of key distance provided by music theorists.

If this empirically derived map of musical keys is valid, and if the interelement associations at the level of tones and chords are mediated through their functions in musical keys as suggested earlier, then the perceived associations between elements should change systematically as the context key is varied. This is the hypothesis tested in the present experiments, which investigate key distance effects on the perceived associations between chords. The experiments were designed to evaluate the specific prediction that the representation of harmonic information about chords depends on the context key in which they are embedded, and that changes in this representation are related to key distance at the highest level of the proposed structural system. The internal representation of relations between chords is expected to undergo lawful changes as a function of the tonality prevailing within the experimental context.

Bharucha and Krumhansl (in press) determined three independent ways in which adding a tonal context affected perceived harmonic relations. These context effects depended on whether the chords were, or were not, contained within the basic set of harmonies of the context key. The results argue against invariant relations at the level of chords; Krumhansl (1979) made a similar argument against invariant relations obtaining for single tones embedded in a tonal context. For both kinds of elements, key membership appears to significantly alter the ways in which the elements are heard in relation to one another. The present experiments test whether the distance between abstract tonal centers is systematically reflected in the magnitude of these context effects, a result that would simultaneously substantiate the psychological importance of both key membership and key distance.

\section{Three Context-Dependent Principles Governing Harmonic Organization}

The three context-dependent principles identified and tested previously (Bharucha \& Krumhansl, in press) govern harmonic organization in the presence of a tonal context. Analogous principles are assumed to hold for the interrelations among single tones, and evidence supporting this assumption will be described as each principle is stated. Each one of these principles is extended here to incorporate effects of key distance, and the more general statements of these principles will be evaluated in the present experiments. As originally stated, the principles characterize the relations among chords in a way that depends on whether the chords were or were not in the context key. In the previous statement, key membership was considered an all-or-none property. The present formulation incorporates key distance by assuming that the degree to which the stated principles hold is a function of the distance between the context key and the keys of which the chords are members. Structural properties governing the harmonic relations between chords are described in this way as systematically moderated by interkey distance.

In the following statements of the three contextdependent principles, we use $d_{K}\left(C_{1}, C_{2}\right)$ to denote the psychological distance between chords, $C_{1}$ and $C_{2}$, when the two chords are heard in succession in the presence of a context key, K. Let CeK mean that the chord, $\mathrm{C}$, is diatonic in that key (a member of the basic set of harmonies). If $\mathrm{C}$ is nondiatonic in the key, $K$ (outside the basic set of harmonies), this is denoted by C\&K.

The first principle is that of contextual identity. It states that when a chord is in the context key it will be perceived as more closely related to itself (less distant from itself) than when it is out of the context key. It should be noted that whereas distances in a metric space must have the property of minimality-that is, the distance between a point and itself must be zerothis property does not necessarily hold for psychological measures of distance or proximity. Using the notation just defined, this principle is written as:

\section{(1) Contextual identity:}

$$
d_{K}(C, C)<d_{K}{ }^{\prime}(C, C),
$$

where $C_{\varepsilon} K$ and $C \notin K^{\prime}$. The psychological distance between an element and itself may be operationally defined as the probability that it is correctly recognized or named; variations in these measures have been noted previously (Krumhansl, 1978; Tversky, 1977). In the present context, we use recognition memory performance as the measure of the psychological distance between a chord and itself. The contextual identity principle predicts that a chord in the context key (for example, the D major chord in the context key of $G$ major) is more likely to be correctly recognized as having been heard previously than if the chord is not in the context key (say, the D major chord is an F major context key). This is be- 
cause the D major chord is in the basic set of harmonies of the key of $G$ major but not $F$ major.

The previous study on recognition memory for chord sequences supported this principle (Bharucha \& Krumhansl, in press). Sequences were more often correctly recognized when all chords were drawn from a single key than when a nondiatonic chord was contained in an otherwise tonal sequence, or when the sequence consisted of a series of randomly selected chords. Similar findings have been obtained for melodic sequences (Dewar, Cuddy, \& Mewhort, 1977; Francès, 1972; Krumhansl, 1979). Dewar et al. (1977) suggested that tonal sequences may contain relational cues that provide information concerning the individual pitches of the sequence.

As stated above, the contextual identity principle assumes that the psychological proximity of a chord to itself will have one of two values, depending on whether the chord is or is not in the context key. We suggest here, however, that this value may show finer gradations, depending on the particular context key, and that this variation may be described as a monotonic function of the distance between the context key and the keys of which the chord is a member. The measure of interkey distance employed is that provided by music theorists; it found some empirical support in the tone-profile study (Krumhansl \& Kessler, 1982) described earlier. The more general statement of the contextual identity principle incorporates this predicted effect of key distance.

Most chords are members of more than one key; let $K(C)$ denote the set of keys of which the chord, $C$, is a member, and let $\delta[K, K(C)]$ denote the average distance between the context key and each of the keys in the set $\mathrm{K}(\mathrm{C})$. This average might possibly be weighted by the relative harmonic importance of the chord in each of the keys of which it is a member. However, there are two reasons we need not be concerned in the present article with the precise formulation of this measure. First, here we used chords from two maximally distant major keys. Second, the context keys in this study were all major keys, which have the property that keys sharing chords are close on the circle-of-fifths (Krumhansl \& Kessler, 1982). Consequently, different formulations of the required average interkey distance would not be expected to affect the orderings of the interkey measure.

The more general form of the contextual identity principle states that the closer the context key is to the keys of which a given chord is a member, the more closely related to itself the chord will be perceived to be. This may be written as:

(1') Contextual identity:

$d_{K}(C, C)$ decreases as $\delta[K, K(C)]$ decreases.
This means that a chord will be less difficult to recognize as the average distance decreases between the context key and the keys of which the chord is a member. For example, a $\mathrm{C}$ major chord, which is a member of $F, C$, and $G$ major keys, will be relatively easy to recognize in a $G$ major context, less frequently recognized in an $A$ major context, and even less frequently recognized in a B major context. (The relations among these keys on the circle of fifths is shown in Figure 1.)

The second principle is contextual distance. It states that two chords are perceived as more closely related to each other when they are both in the context key than when they are not both in the context key. This is written as:

\section{(2) Contextual distance:}

$$
d_{K}\left(C_{1}, C_{2}\right)<d_{K}^{\prime}\left(C_{1}, C_{2}\right),
$$

where $C_{1}, C_{2} \varepsilon K$, and $C_{1}, C_{2} \varangle K^{\prime}$. We have already noted that, in experiments on both single tones (Krumhansl, 1979) and chords (Bharucha \& Krumhansl, in press), the effect of explicitly establishing a tonal context was to strengthen the perceived relatedness among elements in that context key. Moreover, recognition memory performance for melodic sequences (Cuddy et al., 1979; Dowling, 1978) and chord sequences (Bharucha \& Krumhansl, in press) shows frequent confusions between elements in the prevailing key.

The principle of contextual distance may be generalized to specify the effect of varying the distance between the context key and the keys of which both chords are members. Let $K\left(C_{1}, C_{2}\right)$ denote the set of keys of which both $C_{1}$ and $C_{2}$ are members; $\delta[K$, $K\left(C_{1} C_{2}\right)$ ] will stand for the average distance between the context key and those keys. The generalized principle thus states that the perceived distance between chords will decrease as this average distance decreases, written as:

\section{$\left(2^{\prime}\right)$ Contextual distance:}

$$
d_{K}\left(C_{1}, C_{2}\right) \text { decreases as } \delta\left[K, K\left(C_{1}, C_{2}\right)\right] \text { decreases. }
$$

(It is assumed that both $C_{1}$ and $C_{2}$ are members of some key.) To illustrate this principle, consider the $\mathrm{C}$ major and $G$ major chords, which are each members of both the $\mathrm{C}$ major and $\mathrm{G}$ major keys. The contextual distance principle predicts that the closer the context key is to these keys on the circle of fifths (considering only major keys), the stronger the perceived association between these two chords. (Note that contextual identity is simply a special case of contextual distance with $C_{1}=C_{2}$.)

The final principle to be described is contextual asymmetry, which governs the temporal ordering of 
chords. This principle applies to pairs of chords, one of which is in the context key and the other of which is not in the context key. The principle says that two chords in succession will be perceived as more closely related when the first chord is out of the context key and the second chord is in the context key than when the same two chords appear in the reverse temporal order. Formally, this is:

\section{(3) Contextual asymmetry:}

$$
\mathrm{d}_{\mathrm{K}}\left(\mathrm{C}_{1}, \mathrm{C}_{2}\right)<\mathrm{d}_{\mathrm{K}}\left(\mathrm{C}_{2}, \mathrm{C}_{1}\right) \text {, }
$$

where $C_{1} \notin K$ and $C_{2} \in K$. Asymmetries in psychological distances have been noted in a number of domains (Krumhansl, 1978; Rosch, 1975; Tversky, 1977) and have been found to be very characteristic of judgments about musical tones and chords. For example, Bharucha and Krumhansl (in press) found a preference for two-chord sequences that moved from a chord outside the context key to a chord within the key over the reverse temporal order. Similar effects were found in the scaling study of single tones (Krumhansl, 1979). In addition, recognition-memory studies of sequences of tones (Krumhansl, 1979; Dowling \& Bartlett, Note 1) and chords (Bharucha \& Krumhansl, in press) have found frequent confusion errors when a previously heard nondiatonic element was replaced by a diatonic element. These errors were more frequent than when a diatonic element was replaced by a nondiatonic element. Thus, there is considerable support for temporal asymmetries in the psychological distances between different musical elements.

Again the strength of this effect is presumed to be related to the distance between the context key and the keys of which the first and second chords are members. The contextual asymmetry principle may be generalized to take into account the effect of the context key. When two chords are heard in succession in a tonal context, they will be perceived as more closely related: (1) the farther the context key is from the keys of which the first chord is a member, and (2) the closer the context key is to the keys of which the second chord is a member. In our notation, this is:

\section{(3') Contextual asymmetry:}

$$
\begin{gathered}
\mathrm{d}_{K}\left(C_{1}, C_{2}\right) \text { decreases as } \delta\left[K, K\left(C_{1}\right)\right] \text { increases } \\
\text { and } \delta\left[K, K\left(C_{2}\right)\right] \text { decreases. }
\end{gathered}
$$

Consider the $\mathrm{F \#} \mathrm{(Gb)} \mathrm{major} \mathrm{chord} \mathrm{[which} \mathrm{is} \mathrm{in} \mathrm{the}$ keys of $B, F \#(G b)$, and $D b$ major] and the $C$ major chord (which is in the keys of F, C, and G major). The contextual asymmetry principle states that the psychological distance between the $F \#$ major chord and the $\mathrm{C}$ major chord will decrease as the context key moves farther from the keys of which the $F \#$ major chord is a member and closer to the keys of which the $\mathrm{C}$ major chord is a member.

Each of these more general statements of the context-dependent principles assumes that the internal representation of harmonic structure undergoes systematic alterations, depending on the tonality of the context, and that these changes are a function of interkey distances at the level of abstract tonal centers. The context key is presumed to influence interelement associations at both the level of single tones and at the level of chords, although only harmonic relations between chords are explicitly tested here. If the more general principles are substantiated by the present experiments, then we may conclude that invariance at the level of abstract tonal centers (the structure contained within the regular pattern of interkey distances) governs the perceived associations between the individual tones and chords. The perception of musical organization, then, would quite conclusively be shown to be mediated through a system that assigns significance to the musical elements according to their function within different interrelated musical keys. Moreover, invariant properties at the levels of single tones and chords that do not make reference to tonal structure are unlikely to be identified.

\section{EXPERIMENT 1: KEY DISTANCE EFFECTS ON THE SPATIAL REPRESENTATION OF HARMONIC RELATIONSHIPS}

The first experiment investigated how the perceived relations among chords change as a function of the context key. As in the earlier study (Bharucha \& Krumhansl, in press), we employed the set of 14 chords in $\mathrm{C}$ major and in $\mathrm{F \#} \mathrm{major,} \mathrm{with} 7$ chords in each of these two maximally distant major keys. All possible ordered pairs of these chords were presented after a cadence in one of the following three keys: $G$ major, A major, or B major. The chord cadence employed was a IV V I cadence in the designated key, which is a strong instantiator of that key. (The Roman numerals designate the position of the root of the chords in the scale of the key.) As can be seen on the circle of fifths in Figure 1, G major is closely related to $\mathrm{C}$ major (one position away) and distantly related to $\mathrm{F \#} \mathrm{major} \mathrm{(five} \mathrm{positions} \mathrm{away),} \mathrm{A}$ major is moderately distant (three positions away) from both $C$ and $F \#$ major, and $B$ major is close to $F \#$ major (one position away) but distant from $\mathrm{C}$ major (five positions away). The design allowed us to test two of the generalized principles just stated: contextual distance and contextual asymmetry. Since repeating chord pairs were not contained in the experiment, contextual identity was not tested in this experiment, but was tested in the second experiment, which looked at recognition memory for chord sequences. 


\section{Method}

Subjects. Sixteen individuals from the Cornell summer school community were paid $\$ S$ for participating in two experimental sessions totaling $13 / 4$ hours. All listeners had had at least 3 years of instruction on a musical instrument or voice, with an average of 9.2 years. They were currently engaged in musical activities for an average of $4.8 \mathrm{~h}$ per week. Eight reported having taken one or more music theory courses at the college level. All listeners reported having normal hearing, and none reported having absolute pitch.

Apparatus. Chords were digitally computed and were stored on a Hewlett-Packard $1000 \mathrm{~L}$ computer and converted to analog form by a Hewlett-Packard (59303A) digital-to-analog converter. An A.P. Circuit Corporation variable frequency filter (Model AP255-5) eliminated high-frequency noise introduced by the conversion process. Recordings were made on Maxwell UD 35-90 tapes using a Revox A77 tape recorder. The tapes were played back at $7 \frac{1}{2} \mathrm{in} . / \mathrm{sec}(19 \mathrm{~cm} / \mathrm{sec})$ on the same tape recorder through an Ampex AA-620 loudspeaker at approximately 67 dBA soundpressure level.

Stimulus materials. The chords consisted of sine-wave components over a 5-octave range. These component pitches were drawn from the set of equally spaced semitones on a logarithmic scale based on $440 \mathrm{~Hz}$ (A). An amplitude envelope was imposed on the chord components so that the tones at the low and high ends of the 5-octave range tapered off to threshold. This method was based on a technique introduced by Shepard (1964) and described in detail by Krumhansl et al. (1982), who used it to study harmonic relations while minimizing melodic factors. Each chord was sounded for $500 \mathrm{msec}$, with 10 -msec amplitude rise and fall times to reduce onset and off set clicks.

On each trial, a three-chord cadence (IV V I) was presented in one of the three context keys: $\mathrm{G}$ major, A major, or B major. This cadence was followed by the two test chords in succession. The test chords were all possible ordered pairs of nonrepeating chords drawn from the set of 14 chords in $C$ major and $F \#$ major (the IVII chords of each of these keys). The trials were blocked by context key. Each block consisted of a random sequence of 182 trials. There were 10 practice trials before the test trials; these were identical to the last 10 trials of that test block. A pause of approximately $250 \mathrm{msec}$ separated the three successive context chords from each other and the two test chords from each other. A 750-msec pause separated the context chords from the test chords. Between trials, a 4-sec interval allowed the listeners to record their responses. A 500 -msec burst of white noise marked the beginning of each group of 10 trials.

Procedure. The participants were instructed to rate how well the second test chord followed the first test chord in the context of the three-chord cadence preceding the test chords. On a 7-point scale, 1 was designated "follows poorly" and 7 was designated "follows well." The listeners heard the three blocks of trials in different random orders.

\section{Results}

Individual differences. The three $14 \times 14$ matrices of relatedness judgments for each listener from the three context conditions were correlated with the matrices for each of the other listeners. These intercorrelations averaged .381 , and all but 4 of the 120 correlations were significant $(p<.05)$. The hierarchical clustering technique (Johnson, 1967) applied to these intersubject correlations produced a solution with no discernible relationship to music theory background or any other aspect of musical experience. Consequently, the remaining analyses do not distinguish between listeners on the basis of musical training.
Contextual distance. Two separate analyses were performed to determine if the distance between the key in which the chords function (C major or $F \#$ major) and the key of the context (G, A, or B major) affected ratings of chords within each of the two keys. In the first analysis, multidimensional scaling (Kruskal, 1964; Shepard, 1962) was applied to each of the three $14 \times 14$ matrices averaged across subjects and collapsed across the diagonal. The particular program employed was MDSCAL (Kruskal, Note 2), using Stress Formula 1. The results are shown in the bottom three panels of Figure 2 for the $G, A$, and B major context keys. The stress values for these solutions were $.151, .152$, and .162 , respectively. The top three panels show the results obtained in the earlier study (Bharucha \& Krumhansl, in press) for the C major context, no context, and $F \#$ major context conditons. Within each panel, the chords of $\mathrm{C}$ major are located on the left of the configuration and those of F\# major on the right. The chords of these two major keys are clearly separated according to key membership. In addition, the extent to which chords of each key are drawn together depends on the distance of the key of the chords from the key of the context cadence. For example, the chords from $\mathrm{C}$ major are grouped most strongly when the context establishes the key of $\mathrm{C}$ major, followed by $\mathrm{G}$ major, A major (and the no context condition), B major, and finally F\# major. The opposite order is found for the chords from $F \#$ major. Thus, as the context key is moved around the circle of fifths, systematic varia-

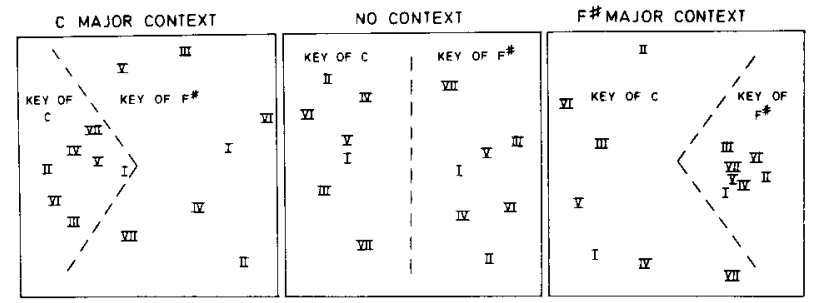

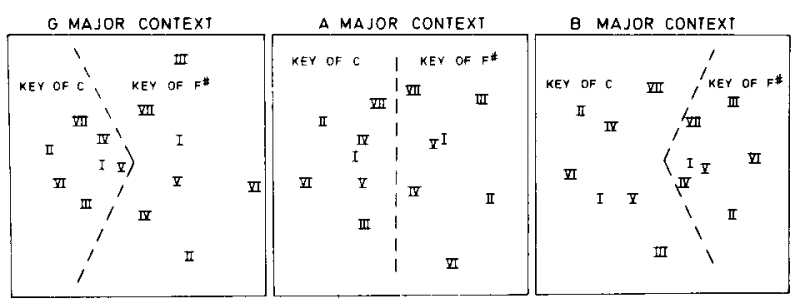

Figure 2. Multidimenslonal scaling solutions of the seven chords from $\mathbf{C}$ major (on the left of each solution) and the seven chords from F* major (on the right of each solution). The top three panels show the solutions obtained by Bharucha and Krumbansl (in press) for the chords presented in a C major context (left), in no context (center), and in an F" major context (right). The bottom three panels show the results obtained in Experiment 1 for the chords in a $G$ major context (left), and A major context (center), and a B major context (right). 
tions in the compactness of the chords in each key occurred in the multidimensional scaling solution.

The second analysis computed for each of the three context keys the average rating given to pairs of chords both of which were in either $\mathrm{C}$ major or $\mathrm{F \#}$ major keys. The means are shown in the top panel of Figure 3. Analysis of variance on these scores supported the contextual distance principle. There was a significant interaction between the key of the chords and the key of the context cadence $[\mathrm{F}(2,30)=52.82$, $\mathrm{p}<.001$ ]. Pairs of chords in $\mathrm{C}$ major were judged to be more similar when the context key was closer to $C$ major on the circle of fifths. The analogous result was also found for pairs of chords in $F \#$ major. There was no main effect of the context key $[F(2,30)$ $<1$, but pairs of chords in $\mathrm{C}$ major were given higher ratings on the average than those in $\mathrm{F \#} \mathrm{major}$ $[F(1,15)=10.44, p<.01]$. In summary, both in terms of the relatedness ratings themselves and the multidimensional scaling solution, the results show a decreasing degree of association between the chords from a single key as a function of the distance between the key of the chords and the key of the context cadence.

Contextual asymmetry. In addition to the effect of key distance on the judgments of pairs of chords from a single key, effects were also found on judgments of pairs of chords from different keys. These pairs are ones in which one chord is a member of the $\mathrm{C}$ major key and the other chord is a member of the

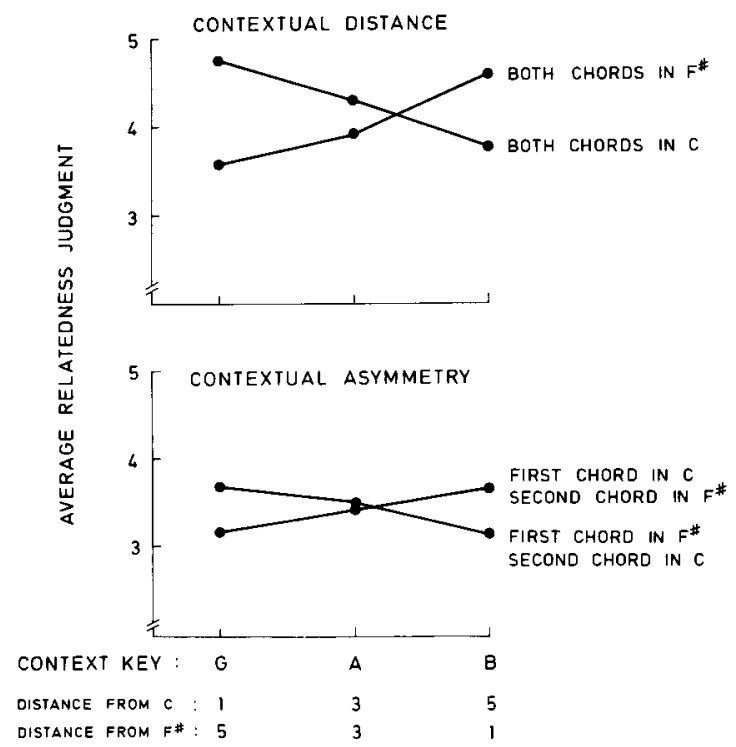

Figure 3. The top graph shows the effect of the contextual distance principle. The average rating given in Experiment 1 to pairs of chords that are either both in $\mathbf{C}$ major or both in $F^{*}$ major is plotted as a function of the context key (G, A, or B major). The bottom graph shows the effect of the contextual asymmetry principle. The average rating given to pairs of chords with one chord in $C$ major and one chord in $F *$ major is plotted as a function of the context key.
F\# major key. There were 49 such pairs of chords, with two temporal orders of each. The average rating for each of the orders in each of the three contexts is shown in the bottom panel of Figure 3. A three-way analysis of variance was computed on the 2 orders, 3 context keys, and the 49 pairs of interkey chords (which was used as the replication factor in the analysis). There was a significant interaction between temporal order and context key $[\mathrm{F}(2,96)=36.08$, $\mathrm{p}<.001]$. As can be seen in Figure 3, as the distance of the context key from $\mathrm{C}$ major increased, the ratings for chord pairs ending on chords in $\mathrm{C}$ major decreased and the ratings for chord pairs ending on chords in F\# major increased. Thus, judgments of pairs of chords from different keys were found to be affected by the nature of the cadence preceding the test chords. There was no main effect of temporal order $[F(1,48)<1]$ or context key $[F(2,96)<1]$.

A derived measure of interkey distance. As mentioned earlier, Krumhansl and Kessler's (1982) application of the profile technique yielded a spatial map of musical keys, which produced the circle of fifths in two dimensions for the major keys. Given the systematic variations found here in ratings of harmonic relations as a function of the context key, it is possible that these judgments may be used to provide convergent evidence for the representation of key distance obtained earlier. To test this, the three $14 \times 14$ half-matrices (for $G, A$, and B major contexts) from this experiment and the two $14 \times 14$ halfmatrices (for $C$ and $F \#$ major contexts) from the study by Bharucha and Krumhansl (in press) were intercorrelated. In other words, the matrix of relatedness ratings for each context was correlated with that for each other context. When multidimensional scaling was applied to the resulting $5 \times 5$ matrix of correlations (between all possible pairs of keys), a solution with zero stress was obtained in one dimension with the keys perfectly ordered according to distance around the circle of fifths, that is, as C, G, A, B, and F\#. Shepard (1974) noted that a circular configuration of $180 \mathrm{deg}$ or less is equivalent in nonmetric multidimensional scaling to a linear solution in one dimension. Consequently, because the musical keys spanned only one half of the circle of fifths, the onedimensional solution was expected. Thus, the present judgments of harmonic relations substantiated the circle-of-fifths dimension of key distance obtained for major keys through the tone profile technique.

Interkey structure independent of context. In addition to the systematic effects of context on the harmonic structure of the 14 chords within the two major keys, the results contained a regular pattern of interrelations among the seven chords of each key independently of the context key. These results replicate earlier findings on within-key harmonic structure (Bharucha \& Krumhansl, in press; Krumhansl et al., 1982) and will be noted only briefly. First, 
within each key the most harmonically significant (I, IV, and V) chords were rated as being most strongly interrelated. Each of the $7 \times 7$ matrices of judgments for the seven chords in $\mathrm{C}$ major and in F\# major in each of the three contexts was analyzed, using the hierarchical clustering technique (Johnson, 1967). In each solution, the I, IV, and V chords clustered first and the less harmonically stable chords joined this cluster later. When the six matrices were averaged, the hierarchical clustering method of this average matrix produced a solution in which the chords clustered in the following order: I, V, IV, VI, II, VII, and III, which is virtually identical to the hierarchical clustering solutions obtained in earlier studies. Thus, the hierarchy of harmonic functions of chords within keys has been found to be a stable structure independent of the context key.

In addition, a regular pattern of asymmetries was found for chord pairs involving one chord within the harmonically stable core (I, IV, V) and one chord outside this core (II, III, VI, VII). In all six cases (chords from either $\mathrm{C}$ or $\mathrm{F} \#$ in each of the three contexts), there was a significant preference for the chord pairs that ended on the chords in the harmonic core over pairs ending on chords outside the core. (Analysis of variance of the two temporal orders for the 12 chord pairs all showed significant asymmetries at the $\alpha=.05$ level.) It should be noted that the chords within the harmonic core are major chords and those outside the core are either minor or diminished chords, so this result may reflect in part the different perceptual effects of the various chord types. These findings replicate the pattern of perceived relations among chords within each key obtained in earlier scaling studies of chords (Bharucha \& Krumhansl, in press; Krumhansl et al., 1982).

\section{Discussion}

The present scaling study of the chords from $\mathrm{C}$ major and $F \#$ major showed key distance effects on the perceived relatedness between these chords. The general statement of the contextual distance principle was substantiated by the finding that the strength of the association between chords in a single key increased as the distance between that key and the key of the context decreased. In addition, the contextual asymmetry principle was confirmed. When one chord was from $\mathrm{C}$ major and the other chord from F\# major, asymmetries that depended on the temporal order of the chords were found to depend on the distance of the context key from $\mathrm{C}$ major and from $\mathrm{F} \#$ major. When the context key was closer to $\mathrm{C}$ major than to F\# major on the circle of fifths, there was a preference for chord pairs ending in a chord from $\mathrm{C}$ major; when the context key was closer to $\mathrm{F \#}$ major, there was a preference for sequences ending on chords from F\# major. Finally, intercorrelations between the proximity matrices for the three context keys employed in this study and the two context keys of the earlier scaling study (Bharucha \& Krumhansl, in press) recovered the circle-of-fifths dimension obtained in the tone profile experiment (Krumhansl \& Kessler, 1982). This provides convergent evidence for the measure of key distance derived in that earlier study.

These findings support the hypothesis that harmonic relations between chords are mediated by a process that interprets their functions in musical keys. Depending on the key instantiated within the experimental context, the perceived strength of association between chords changed, and these changes were found to be a systematic function of the distance between the key from which the chords were drawn ( $C$ or F\# major) and the context key ( $G, A$, B), as measured on the circle-of-fifths. Harmonic relations are seen to be very context dependent and, moreover, do not depend simply on key membership in an all-or-none fashion, but are sensitive to the distances between keys at the highest level of the proposed three-leveled structure.

\section{EXPERIMENT 2: KEY DISTANCE EFFECTS ON RECOGNITION OF HARMONIC SEQUENCES}

The second experiment tested the generality of the key-distance effects found in Experiment 1. The dependent measure used in this experiment was the accuracy of recognition memory for sequences of chords. If, as suggested above, the effect of varying the context key is to restructure the internal representation of harmonic relations among chords in a way that depends systematically on key distance, then these variations should be reflected in the frequency of confusions between chords, not just in judgments of the degree of relatedness between chords.

In this experiment, listeners heard pairs of sevenchord sequences that were either identical or different from each other in terms of one chord. The listener's task was to judge whether or not the two sequences were the same. When the sequences were different, the chord in the fourth position in the first sequence was replaced by another chord in the fourth position of the second sequence. Thus, we will refer to the chords in the fourth positions as the target chords. The target chords were drawn from the set of 12 major or minor chords from the keys of $C$ major and $F \#$ major (I-VI from C and I-VI from F\#).

The target chords were embedded in tonal sequences that instantiated one of the following context keys: C major, $\mathrm{G}$ major, A major, or B major. Each sequence began with the tonic (I) chord of the context key in the first serial position and ended with a dominant(V)-tonic(I) authentic cadence in serial positions 6 and 7 . The remaining serial positions (the 
second, third, and fifth) were filled by chords drawn from the set consisting of the II, III, IV, V, and VI chords of the context key. Diminished seventh chords (VII) were not used in the context sequences or as target chords because they are relatively unstable harmonically and are of a different type from the other chords, which are all either major or minor. An F\# major context was not used, because, due to its position opposite to $\mathrm{C}$ major on the circle of fifths, it would essentially constitute a replication of the $C$ major context.

In these tonal sequences, the sequential ordering of chords conformed to Piston's (1962, p. 18) rules of harmonic progression. However, these rules were not applied to the target chord, which was drawn from the key of either $C$ major or F\# major, and was therefore frequently outside the key of the context. In addition to the tonal context sequences in one of the four keys (C major, $G$ major, A major, or B major), random sequences were also included. The chords in these sequences were selected randomly from the set of all major and minor chords, subject to the constraint that they be comparable to the tonal sequences in terms of the types of chords employed (major or minor) and the number and position of repeated chords. The accuracy of recognition-memory performance in this design can be used as a test of the three generalized contextual properties given earlier: contextual identity, contextual distance, and contextual asymmetry.

\section{Method}

Subjects. Fifteen individuals from the Cornell community were paid $\$ 16$ for participating in four experimental sessions totaling $41 / 2 \mathrm{~h}$. They had received instruction on a musical instrument or in voice for an average of 9.0 years, and currently were engaged in musical activities for an average of $7.5 \mathrm{~h}$ per week. Six of the 15 listeners had taken at least one music theory course at the college level. All reported having normal hearing, and none reported having absolute pitch.

Apparatus and Stimulus materials. The chords were synthesized using the same apparatus as in Experiment 1 and according to the same specifications. Approximately $250 \mathrm{msec}$ separated chords within a sequence, and a 750 -msec pause separated the first (standard) sequence from the second (comparison) sequence. Between trials, the subjects had $4 \mathrm{sec}$ during which to indicate their responses. A 500 -msec burst of white noise signaled the beginning of each group of 10 trials.

Each trial consisted of two seven-chord sequences, which were either identical or different in the fourth serial position. The structure was as described earlier. Altogether, there were a total of 660 "different" trials and 360 "same" trials. The "different" trials consisted of all possible ordered pairs (132) of nonrepeating chords from the set of 12 chords from $C$ major and $F \#$ major (the I-VI chords in each of these keys). Each one of these pairs was embedded in each of the five types of context sequences: random, or tonal in $\mathrm{C}$ major, $\mathrm{G}$ major, $\mathrm{A}$ major, or $\mathrm{B}$ major. This yielded 660 "different" trials. On "same" trials, 1 of the 12 chords in $\mathrm{C}$ or $\mathrm{F} \#$ major was contained in the fourth serial position of both the standard and comparison sequences, which again might be either random or tonal in one of the four context keys. Six replications gave a total of 360 "same" trials. These trials were randomly intermixed and divided into eight blocks of trials, which were heard by the listeners in different random orders with two blocks per session.

Procedure. The listeners were instructed to judge whether the two sequences on each trial were the same or different. A 4-point scale was used for this response $(-2=$ "very sure same," $-1=$ "same but not sure," 1 = "different but not sure," 2 = "very sure different"). The participants were told that there were roughly twice as many "different" trials as "same" trials, and that if the standard and comparison sequences were different it would be the fourth chord that was changed. The first session began with 10 randomly selected practice trials.

\section{Results}

The responses were first dichotomized and scored as correct or incorrect. Analyses were then done separately for "same" trials, for "different" trials in which the target chords were from the same key (both from C major or both from F\# major), and for "different" trials in which the target chords were from different keys (one from $C$ major and one from $F \#$ major). These analyses correspond to the three context-dependent principles stated earlier.

Contextual identity. The average probability of a correct "same" response for trials with target chords from $\mathrm{C}$ or $\mathrm{F \#} \mathrm{major} \mathrm{in} \mathrm{each} \mathrm{of} \mathrm{the} \mathrm{four} \mathrm{tonal} \mathrm{context}$ sequences is shown in the top panel of Figure 4. This figure shows that the probability of a correct response increased as the distance between the tonal context key (C, G, A, or B major) and the key of the target chord (C or F\# major) decreased. An analysis of variance done on the percent correct scores for the four tonal conditions showed this result as the significant interaction between the key of the tonal context and the key of the target chord $[F(3,42)=41.711$, $\mathrm{p}<.001]$. Thus, the recognition scores supported the contextual identity property. Both the main effect of the key of the target chords and the main effect of the context key were significant. On the average, performance was higher for chords from $\mathrm{C}$ major than for chords from $F \#$ major $[F(1,14)=30.975, p<.001]$. This may be accounted for by the fact that the tonal context keys were generally closer to $C$ major on the circle of fifths than they were to $F \#$ major. The main effect of context key $[F(3,42)=7.252, p<.001]$ reflected the finding that performance tended to be better when the context key was closer to either one of the keys of the target chords than when it was relatively distant from both.

Performance on the random sequences was generally high and showed no significant difference between target chords from $\mathrm{C}$ major and $\mathrm{F} \#$ major. The probability of a correct response was .84 for target chords from $\mathrm{C}$ major and .88 for target chords from F\# major. This generally high performance on "same" trials in the random sequence condition may, however, simply reflect a bias to say "same" when the sequences are random, since performance on "different" trials with random sequences was relatively low, as will be described later. 


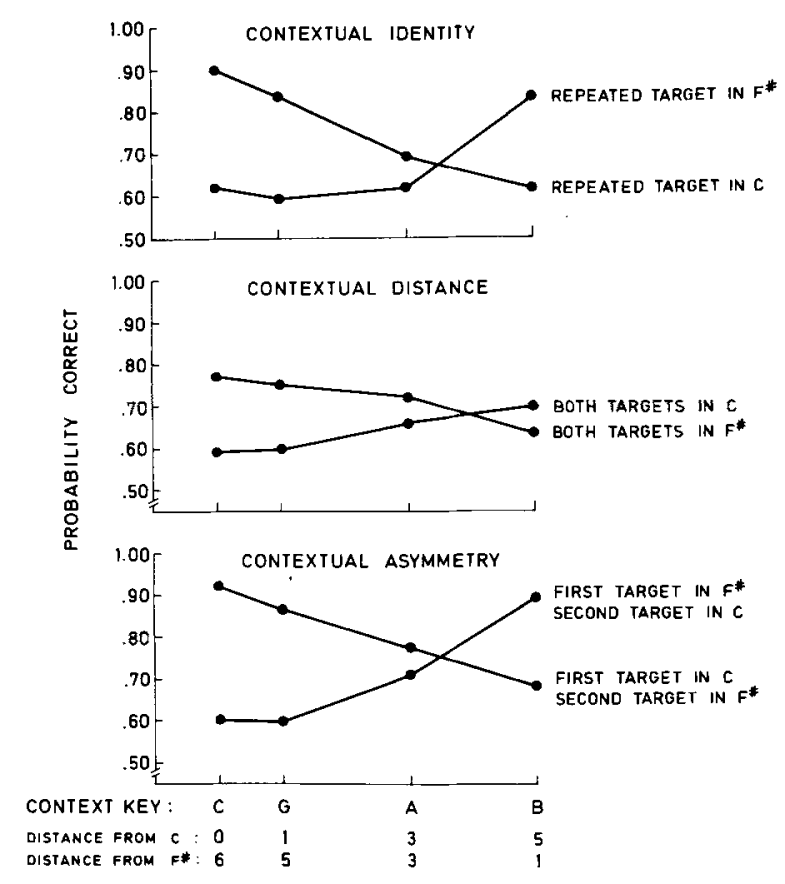

Figure 4. The top graph shows the effect of the contextual Identity principle. The probability of a correct "same" judgment for target chords in C and $F$ " major keys is plotted as a function of the key of the sequence as a whole (C, G, A, or B major). The center graph shows the effect of the contextual distance principle. The probability of a correct "different" Judgment for target chords both in C or in F" major is plotted as a function of the key of the sequence. The bottom graph shows the effect of the contextual asymmetry principle. The probabllity of a correct "different" Judgment when one target chord is in $C$ major and the other target chord is in F* major is plotted as a function of the key of the sequence.

Contextual distance. The next analysis was performed on "different" trials in which a target chord from $\mathrm{C}$ or $\mathrm{F \#} \mathrm{major} \mathrm{in} \mathrm{the} \mathrm{standard} \mathrm{sequence} \mathrm{was} \mathrm{re-}$ placed by another chord from the same key in the comparison sequence. The average probability of a correct "different" response for these trials is shown in the middle panel of Figure 4 as a function of the key of the tonal contexts. The contextual distance principle was confirmed by the significant interaction between the key of the target chord ( $C$ or $F \#$ major) and the key of the tonal context $(C, G, A$, or $B$ major) $[F(3,42)=6.774, p<.001]$. More confusions occurred between target chords from the same key when the key of the context was closer to that key on the circle of fifths. Again, there was a significant main effect of the key of the target chord $[F(1,4)=29.945$, $\mathrm{p}<.001$, with an advantage for target chord pairs from $F \#$ major. This may be accounted for by the selection of tonal context keys that are generally closer to $\mathbf{C}$ major than to $\mathrm{F} \#$ major on the circle of fifths, causing more confusions overall between chords from C major. Here, however, there was no main effect of the key of the tonal contexts $[F(3,42)<1]$.
Generally poor performance was found for the random sequences. On these trials, the average probability of a correct response was .50 for target chords from $C$ major and .60 for target chords from $F \#$ major. [This difference was marginally significant$t(14)=2.473, p<.05$ ]. As suggested earlier, the generally low performance on "different" trials for random sequences and the generally high performance on "same" trials in this condition may simply reflect a "same" bias for random sequences.

Contextual asymmetry. The final analysis was performed on "different" trials in which the target chord in the standard sequence was replaced by a chord from a different key in the comparison sequence. These trials are ones in which the first sequence contained a chord from $\mathrm{C}$ major and the second a chord from $F \#$ major, or the opposite. The average probability of a correct response for these trials is shown in the bottom panel of Figure 4 as a function of the key of the sequence. This figure shows that more confusion errors occurred when the context key was closer to the key of the second target chord than to the key of the first target chord. The significant interaction between the key of the second target chord (with the first chord being from the other key) and the key of the context sequence $[F(3,42)=63.378, p<.001]$ confirmed the contextual asymmetry principle. The main effect of the key of the second target chord was also significant $[F(1,42)=42.391, p<.001]$. More confusion errors occurred when the second target chord was from $\mathrm{C}$ major than when it was from $F \#$ major, again possibly reflecting the selection of context keys that are generally closer to $\mathrm{C}$ major than to $\mathrm{F} \#$ major. The main effect of the key of the context sequence was not significant $[F(3,42)=2.406, p>.05]$.

Finally, performance on trials with random sequences was again low. Changes from chords from $\mathrm{C}$ major to chords from $F \#$ major were detected with a probability of .55 , changes from chords from $F \#$ major to chords from $C$ major were detected with a probability of .53 , and these values did not differ statistically.

\section{Discussion}

Recognition memory for chords was found to be affected by the relationship between the key of the target chord and the key of the sequence in which it was embedded. The target chord was more frequently correctly recognized when the context key was closer to the key in which that chord functions. In addition, the number of confusions between different target chords from the same key was found to increase as the distance of the context key from the key of the target chords decreased. Finally, systematic effects of key distance were found on the probability of confusing chords from different keys. More confusions resulted when the context key was 
closer to the key of the target chord in the second, rather than the first, sequence. These results support each of the three contextual principles stated earlier in their general form: contextual identity, contextual distance, and contextual asymmetry.

\section{GENERAL DISCUSSION}

The perceived relations among chords were found in these two experiments to change with the tonal context in which they were embedded. These changes depended systematically on the distance between the context key and the keys of which the chords are members, with this distance being that between keys at the level of abstract tonal centers. Evidence for these alterations in the internal representation of harmonic information was found in two quite different tasks: direct judgments of the degree of relatedness between chords and recognition memory for chords. The context key affected the recognition accuracy of chords and the probability that a chord was confused with another chord in the same key or in a different key. These confusion errors were mirrored by direct ratings of the musical relatedness between chords, with higher ratings correlated with more confusion errors. Thus, the internal representation of harmonic structure in music was found to be strongly context dependent. These results can be understood by assuming that listeners interpret chords in terms of their functions in abstract tonal centers or keys.

More importantly, the finding that these alterations were predictable from the distances between abstract tonal centers provides strong evidence for the internalization of musical structure at the level of musical keys. Very regular differences in relatedness judgments and memory performance were found as the context key was varied around the circle of fifths. In fact, the circle-of-fifths dimension was recovered by intercorrelating the matrices of relatedness judgments for the different context keys. This provides convergent evidence for the measure of interkey distance obtained through the tone profile technique for major keys (Krumhansl \& Kessler, 1982). Similar effects on harmonic relations would presumably be found as a function of the distances between major and minor keys and between different minor keys. Thus, although perceived associations among chords were not found to be invariant across contexts, these alterations may be accounted for by structure at the level of musical keys.

Key distance has also been found to affect recognition memory for transposed melodic sequences that are first played in one key and then played in a different key. For example, Cuddy et al. (1979) showed that melodies transposed to the dominant key (one position around the circle-of-fifths) were easier to recognize than melodies transposed to the key of the tritone (six positions around the circle of fifths). Bartlett and Dowling (1980) found that, although recognition performance varied with the musical experience and age of the listeners and the familiarity of the melody, none of these factors interacted with the magnitude of the key-distance effect, which was present under all conditions. In these earlier studies, key distance was in general confounded with the number of tones shared by the original and transposed melodies (which is necessitated by the fact that closer keys share more scale notes, as discussed earlier). This suggests that the key-distance effects obtained in these earlier studies may depend on the number of repeated tones and not on the relationship between the tonal centers, that is, not on key distance per se. However, Bartlett and Dowling's (1980) analysis of the individual sequences showed only a weak association between the number of repeated tones and recognition performance, and they suggested that, although this factor may have had an influence, their results might better be interpreted in terms of an internal schema of tonal structure (Dowling, 1978). The present results showing keydistance effects on harmonic relations would seem to further support the latter interpretation of these transposition studies. Thus, the processing of both melodic and harmonic information appears to make reference to an internalized system of interrelated tonal centers.

Key distance effects were also found by Krumhansl and Kessler (1982). In that study, the tone profile technique was used not only to obtain the spatial map of musical keys described earlier, but also to trace how the sense of key develops and changes over time during well-structured harmonic sequences. A number of chord sequences consisting of nine chords each were employed. After the first chord of each sequence, ratings of each of the 12 pitches of the chromatic scale were obtained; this gave a profile of ratings for sequences of length 1 . Rating profiles were then obtained after the first two chords of each sequence. This process was continued until profiles were obtained for sequences of lengths 1 through 9 . These profiles were correlated with the profiles for the 24 major and minor keys that came from the strong key-defining elements (scales, chords, and chord cadences). These correlations provided a measure of the strength of all possible key interpretations at every point in time during the sequences.

The results of that study showed that listeners integrate the harmonic roles of the chords in different keys over successive chords, developing a sense of key that may be altered as additional chords are sounded. Most relevant to the present discussion were the differences found between the sequences that modulated (changed) between closely related keys and those that modulated between more dis- 
tantly related keys. For the close modulations, as soon as a chord in the new key was sounded the sense of the new key was stronger than implied by that single chord in isolation. For the distant modulations, the sense of the new key was achieved only later in the sequence. Thus, key distance apparently affects the ease with which modulations between keys are assimilated by listeners. In addition, the prevailing key tended to suppress the sense of distantly related keys below the level predicted by the individual chords in isolation.

In sum, a number of studies indicate that structure at the level of musical keys has been internalized by listeners. In the two experiments reported here, the internal representation of harmonic information, that is, the perceived relations among the individual chords, was found to undergo significant changes in a way that depended on the broader tonal framework in which the chords were embedded. These systematic changes as a function of the distance between the key or keys in which the chords function and the context key were summarized by three principles: contextual identity, contextual distance, and contextual asymmetry. Stated in their general form, these principles describe the effect of varying the tonal context on the psychological distance between chords in either the same or different keys. A number of results on the perceptual processing of melodies suggest that analogous principles may also apply to the level of single tones.

The present article provides a description of the listener's knowledge of harmonic structure and specifies the changes that occur in this representation as the tonal context is varied. Analyses of this kind indicate that listeners possess an articulated system of knowledge about the harmonic functions of the individual chords, and an appreciation of the relations among different musical keys at the level of abstract tonal centers. These internalized structures influence the way in which the individual musical elements are encoded and remembered, and allow the listener to apprehend essential organizational features during music perception.

\section{REFERENCE NOTES}

1. Dowling, W. J., \& Bartlett, J. C. Assimilation of brief atonal melodies to tonal prototypes: Asymmetrical effects on judgment. Paper presented at the meeting of the Psychonomic Society, Philadelphia, November 1981.

2. Kruskal, J. B. How to use MDSCAL, a program to do multidimensional scaling and multidimensional unfolding (Version 4). Murray Hill, N.J: Bell Laboratories, 1968.

\section{REFERENCES}

Bartlett, J. C., \& Dowling, W. J. Recognition of transposed melodies: A key-distance effect in developmental perspective. Journal of Experimental Psychology: Human Perception and Performance, 1980, 6, 501-515.

Bharucha, J., \& Krumhansl, C. L. The representation of harmonic structure in music: Hierarchies of stability as a function of context. Cognition, in press.

Cuddy, L. L., Cohen, A. J., \& Miller, J. Melody recognition: The experimental application of musical rules. Canadian Journal of Psychology, 1979, 33, 148-157.

Dewar, K. M., Cuddy, L. L., \& Mewhort, D. J. K. Recognition memory for single tones with and without context. Journal of Experimental Psychology: Human Learning and Memory, 1977, 3, 60-67.

Dowling, W. J. Scale and contour: Two components of a theory of memory for melodies. Psychological Review, 1978, 85, 341-354.

Francess, R. La perception de la musique (2nd ed.). Paris: Librarie Philosophique J. Vrin, 1972.

Johnson, S. C. Hierarchical clustering schemes. Psychometrika, 1967, 32, 241-254.

KrumhansL, C. L. Concerning the applicability of geometric models to similarity data: The interrelationship between similarity and spatial density. Psychological Review, 1978, 85, 445-463.

Krumhansl, C. L. The psychological representation of musical pitch in a tonal context. Cognitive Psychology, 1979, 11, 346-374.

Krumhansl, C. L., Bharucha, J., \& Kessler, E. J. Perceived harmonic structure of chords in three related musical keys. Journal of Experimental Psychology: Human Perception and Performance, 1982, 8, 24-36.

Krumhanst, C. L., \& Keil, F. C. Acquisition of the hierarchy of tonal functions in music. Memory and Cognition, 1982, 10, 243-251.

Krumhanst, C. L., \& Kessler, E. J. Tracing the dynamic changes in perceived tonal organization in a spatial representation of musical keys. Psychological Review, 1982, 89, 334-368.

Krumhansl, C. L., \& ShePard, R. N. Quantification of the hierarchy of tonal functions within a diatonic context. Journal of Experimental Psychology: Human Perception and Performance, 1979, 5, 579-594.

KRUSKaL, J. B. Nometric multidimensional scaling: A numerical method. Psychometrika, 1964, 29, 28-42.

Piston, W. Harmony (3rd ed.). New York: Norton, 1962.

Rosch, E. Cognitive reference points. Cognitive Psychology, $1975,7,532-547$.

Shepard, R. N. The analysis of proximities: Multidimensional scaling with an unknown distance function. I \& II. Psychometrika, 1962, 27, 125-140, 219-246.

Shepard, R. N. Circularity in judgments of relative pitch. Journal of the Acoustical Society of America, 1964, 36, 2346-2353.

SHEPARD, R. N. Representation of structure in similarity data: Problems and prospects. Psychometrika, 1974, 39, 373-421.

Tvensky, A. Features of similarity. Psychological Review, 1977, 84, 327-352.

(Manuscript received March 8, 1982; revision accepted for publication May 5, 1982.) 